\title{
Differences in Clinical Features and Computed Tomographic Findings between Embolic and Non-Embolic Acute Ischemic Stroke: A Quantitative Differential Diagnosis
}

\author{
Kentaro TaKano*,**, Takenori Yamaguchi*, Kazuo Minematsu*, Tohru SawadA* and Teruo OmaE*
}

A diagnosis based on the presumed mechanism of stroke onset is useful for management strategies in acute ischemic stroke. Ninety-two patients with embolic (cardiac or artery-to-artery) and 107 with non-embolic (thrombotic or hemodynamic) stroke were diagnosed on strict cerebral angiographic criteria alone. To clearly discriminate between these two groups, the neurological and computed tomographic (CT) findings were then compared. Rapidity of onset, vomiting, urinary incontinence, level of consciousness, cervical bruit, anisocoria, tongue deviation, sensory disturbance, and CT findings (location of hypodense area, findings of brain edema and hemorrhagic transformation) were discriminatory factors between the two groups $(p<0.01)$. According to these 11 items, we prepared a numerical table for quantitative differential diagnosis. A diagnostic accuracy of $\mathbf{9 8 . 9 \%}$ for embolic and $\mathbf{8 7 . 9 \%}$ for non-embolic stroke in internal verification, and $90.0 \%$ and $82.9 \%$, respectively, in external verification was observed. The differences in clinical features and CT findings between embolic and non-embolic stroke may reflect the pathophysiological mechanisms of the occlusive process of cerebral artery as well as the extent and severity of ischemia.

(Internal Medicine 37: 141-148, 1998)

Key words: cerebral angiography, cerebral embolism, cerebral thrombosis, discriminatory analysis, multivariate analysis, quantification theory

\section{Introduction}

Acute stroke remains a challenge for stroke neurologists. Neuroprotective and thrombolytic agents are currently being investigated regarding efficacy and safety in acute ischemic stroke. To achieve this goal, accurate clinical diagnosis should be necessary. Acute ischemic stroke can be classified not only into clinical categories, such as cardioembolic, atherothrombotic and lacunar stroke, but also by pathophysiological mechanism, i.e. embolic, thrombotic, or hemodynamic (1). Differentiation of embolic stroke from thrombotic or hemodynamic stroke is particularly important (2-5), because the strategies for management during the acute stage differ, based on the mechanisms of stroke onset rather than clinical subtypes. Subcategorization based on pathophysiological mechanisms can be more valuable in hyperacute ischemic stroke (e.g. regarding an application of thrombolytic therapy). However, differentiation of the pathophysiological mechanisms during the acute stage is often diffi- cult. Clinical diagnostic criteria for embolic stroke have been proposed, mainly for cardioembolic stroke (1-7), whereas the characteristics, severity and treatment appear somewhat different between cardiogenic and artery-to-artery brain embolism (8). There are no useful clinical diagnostic criteria for nonembolic stroke, which has different mechanisms, thrombotic and hemodynamic, however, there have been a few reports suggesting differences in the clinical features according to pathogenetic and pathophysiological mechanisms among embolic and thrombotic or hemodynamic stroke (2-5).

Neuroimaging has been revolutionized over the last two decades, and recent advances of magnetic resonance imaging technology, particularly diffusion-weighted imaging, allow investigators to depict ischemic lesions in vivo during very early stages of acute ischemic stroke (9-14). Computed tomography (CT), however, can be still useful in hyperacute ischemic stroke for the following reasons; 1) to identify hemorrhagic and ischemic stroke accurately, particularly before thrombolytic

From *the Cerebrovascular Division, Department of Medicine, National Cardiovascular Center, Osaka and **the Neurovascular Research Center, National Fukuoka-Higashi Hospital, Fukuoka

Received for publication June 9, 1997; Accepted for publication October 31, 1997

Reprint requests should be addressed to Dr. Kentaro Takano, the Neurovascular Research Center, National Fukuoka-Higashi Hospital, Chidori 1-1-1, Koga, Fukuoka 811-3195 
treatment in hyperacute ischemic stroke, 2) it is simple, fast and available in all emergency hospitals, and 3) allows us to suggest embolic or non-embolic mechanisms when CT findings are typical as previously reported (15-19).

If simple, accurate and reliable clinical diagnostic criteria, such as a numerical table, for pathophysiological mechanisms of acute ischemic stroke were available, it would be of great use in clinical practice (20-24). To try and achieve this goal, it is necessary to clarify the differences in the clinical features and the neuroradiological findings between embolic and non-embolic stroke. A numerical table prepared by a statistical approach could lead to enhanced diagnostic accuracy, but unfortunately most numerical tables are not statistically derived. We attempted to determine the characteristic clinical features of embolic and thrombotic or hemodynamic stroke, and investigated the possibility of clinical differentiation by quantitative diagnosis.

\section{Subjects and Methods}

\section{Patients selection}

The subjects consisted of 435 consecutive ischemic stroke patients with the internal carotid arterial distribution, who were admitted to the Stroke Care Unit of the National Cardiovascular Center within seven days after onset between May 1978 and January 1989. During this period, initial (within seven days after the onset of stroke) and follow-up cerebral angiographies (CAG) were performed in 377 patients (86.7\%). For the 377 patients, we attempted to determine the pathophysiological mechanisms of acute ischemic stroke, such as embolic, thrombotic or hemodynamic on the basis of CAG findings alone (25).

Subjects were selected based on the following CAG criteria alone. Patients showing an embolus shadow, which is a filling defect with trimming, in the initial CAG or reopening of a previously occluded cerebral artery in a follow-up CAG wtihout significant arteriosclerotic changes (wall irregularity) were diagnosed as embolic stroke (EM) (26-28). Absence of arterial occlusion on the initial CAG, which implys an early reopening of the embolic occlusion was not used for criteria of EM. Because thrombotic and hemodynamic strokes based on atherosclerotic occlusive disease are occasionally difficult to differentiate by CAG alone, we assigned patients with a thrombotic or hemodynamic stroke to the non-embolic stroke (NE) category. Thus, patients with cardioembolic stroke and atherothrombotic stroke due to artery-to-artery embolism were categorized as EM, while the remaining atherothrombotic stroke patients due to thrombotic and hemodynamic mechanisms were NE. Criteria for the angiographic diagnosis of $\mathrm{NE}$ by $\mathrm{CAG}$ included an occlusion of a major cerebral artery associated with atherosclerotic changes (wall irregularity), i.e. stump or tapering occlusion at the origin of internal carotid artery (ICA) or the proximal stem (origin) of the middle cerebral artery (MCA) $(3,29)$. Occlusion of the MCA branch with marked arterial wall irregularity was also considered as atherosclerotic in nature (29). No embolus shadow in the initial CAG or reopening phenomenon on a follow-up CAG was included for NE. Table 1 summarizes
Table 1. Angiographic Findings in Embolic Stroke and NonEmbolic Stroke

\begin{tabular}{|c|c|c|}
\hline & $\begin{array}{c}\text { Embolic } \\
\text { stroke } \\
(n=92)\end{array}$ & $\begin{array}{c}\text { Non-embolic } \\
\text { stroke } \\
(n=107)\end{array}$ \\
\hline \multicolumn{3}{|l|}{ Site of occlusion } \\
\hline ICA occlusion & $26(28.3)$ & $44(41.1)$ \\
\hline proximal (extracranial) & 4 & 44 \\
\hline distal (intracranial) & 22 & 0 \\
\hline MCA stem occlusion & $23(25.0)$ & $46(43.0)$ \\
\hline MCA branch occlusion & $30(32.6)$ & $10(9.3)$ \\
\hline Multivessel occlusion & $13(14.1)$ & $7 \quad(6.5)$ \\
\hline \multicolumn{3}{|l|}{ Specific findings } \\
\hline Embolus shadow with reopening & $20(21.7)$ & $0 \quad(0)$ \\
\hline Embolus shadow without reopening & $21(22.8)$ & $0 \quad(0)$ \\
\hline Reopening without embolus shadow & $51(55.4)$ & $0 \quad(0)$ \\
\hline
\end{tabular}

Numbers in parentheses are percentages, ICA: internal carotid artery, MCA: middle cerebral artery.

the angiographic findings of the two groups. One hundred and ninety-nine patients were included in this study, 92 cases with EM (average 62.1 \pm 11.7 years, 43 men and 49 women) and 107 with NE (average $63.8 \pm 9.9$ years, 81 men and 26 women). In EM, 71 of 92 (77.2\%) cases showed complete reopening of previously occluded cerebral arteries, while the remaining 21 cases (22.8\%) without re-opening were diagnosed as EM based on embolus shadow. For NE, $84.1 \%$ of patients showed a major cerebral arterial occlusion, while in EM $32.6 \%$ and $14.1 \%$ were found to have MCA branch occlusion and multi-vessel occlusion, respectively. Brain CT and the clinical features were not considered at all in the patients selection.

\section{Variables used for comparison}

The incidence for each category of the temporal profile, physical findings and neurological findings were retrospectively analyzed (Tables 2, 3), as derived from the patients' clinical records. The results of routine laboratory examinations on admission were also studied (Table 4). To compare the CT findings between EM and NE, the location and configuration of hypodense areas and the presence of brain edema or hemorrhagic transformation were examined on the initial CT (Table 5) as described in previous reports $(15-18,30-32)$. Initial CTs were performed on the average 4.0 days after onset of EM, and 4.1 days in NE. Infarct index was also calculated as a ratio of the hypodense area to the homolateral hemispheric area, and expressed in percent (3). These analyses were performed without CAG information.

\section{Quantitative differentiation table}

Values are expressed as mean \pm SD. Statistical analyses were performed by a chi-square test, two-tailed unpaired $t$-test, and Hayashi's quantification theory group II $(33,34)$. A $p$-value 
Embolic Score in Acute Ischemic Stroke

Table 2. Physiological Findings and Temporal Profile

\begin{tabular}{lccc}
\hline & $\begin{array}{c}\text { Embolic stroke } \\
(\mathrm{n}=92)\end{array}$ & $\begin{array}{c}\text { Non-embolic stroke } \\
(\mathrm{n}=107)\end{array}$ & $\mathrm{p}$-value \\
\hline Body weight $(\mathrm{kg})$ & $52.7 \pm 1.0$ & $54.8 \pm 1.0$ & $\mathrm{NS}$ \\
Respiration rate $\left(\mathrm{min}^{-1}\right)$ & $18.4 \pm 0.4$ & $17.0 \pm 0.3$ & $\mathrm{p}<0.01$ \\
Blood pressure $(\mathrm{mmHg})$ & & & \\
Systolic & $146.6 \pm 25.6$ & $162.7 \pm 25.1$ & $\mathrm{p}<0.01$ \\
Diastolic & $85.1 \pm 14.8$ & $89.6 \pm 14.7$ & $\mathrm{p}<0.01$ \\
Previous TIA & & & \\
Total & $9(9.8)$ & $24(22.4)$ & $\mathrm{p}<0.01$ \\
Stereotype & 4 & 22 & \\
Multivessels & 5 & 2 & \\
Rapidity of onset & & & \\
Abrupt & $66(71.7)$ & $10(9.3)$ & \\
Insidious & $3(3.3)$ & $34(31.8)$ & \\
Stepwise & $4(4.3)$ & $48(44.9)$ & \\
$\quad$ Unknown & $19(20.7)$ & $15(14.0)$ & \\
Time of onset & & & $\mathrm{p}<0.01$ \\
Active & $64(69.6)$ & $56(52.3)$ & \\
At morning & $6(6.5)$ & $41(38.3)$ & \\
During sleep & $15(16.3)$ & $8(7.5)$ & \\
Unknown & $7(7.6)$ & $2(1.9)$ & \\
\hline
\end{tabular}

Numbers in parentheses are percentages. Statistical analyses are chisquare test and two-tailed unpaired $t$-test. TIA: transient ischemic attack, NS: not significant.
Table 4. Laboratory Examinations in Embolic Stroke and NonEmbolic Stroke

\begin{tabular}{lccc}
\hline & $\begin{array}{c}\text { Embolic } \\
\text { stroke } \\
(\mathrm{n}=92)\end{array}$ & $\begin{array}{c}\text { Non-embolic } \\
\text { stroke } \\
(\mathrm{n}=107)\end{array}$ & $\begin{array}{c}\text { Normal } \\
\text { range }\end{array}$ \\
& $9.4 \pm 3.1$ & $8.9 \pm 2.6$ & $4.0-9.0$ \\
\hline Leukocyte $\left(10^{3} / \mu \mathrm{l}\right)$ & $451.0 \pm 54.1$ & $467.5 \pm 67.3$ & $420-550$ \\
Erythrocyte $\left(10^{4} / \mu \mathrm{l}\right)$ & $41.7 \pm 5.0$ & $43.3 \pm 5.9$ & $36.0-54.0$ \\
Hematocrit $(\%)^{\mathrm{c}}$ & $18.8 \pm 7.5$ & $21.0 \pm 6.6$ & $15.0-35.0$ \\
Thrombocyte $\left(10^{4} / \mu \mathrm{l}\right)$ & $138.1 \pm 3.5$ & $138.5 \pm 4.3$ & $138-145$ \\
Serum natrium $(\mathrm{mEq} / l)$ & $3.9 \pm 0.5$ & $3.8 \pm 0.4$ & $3.4-4.9$ \\
Serum potassium $(\mathrm{mEq} / l)$ & $7.4 \pm 0.8$ & $7.2 \pm 0.7$ & $6.5-8.2$ \\
Total protein $(\mathrm{g} / \mathrm{dl})$ & $138.6 \pm 39.5$ & $129.5 \pm 47.3$ & $65-110$ \\
Fasting blood sugar $(\mathrm{mg} / \mathrm{dl})$ & $35.1 \pm 17.6$ & $30.4 \pm 21.6$ & $0-40$ \\
GOT $(\mathrm{U} / l)$ & $182.5 \pm 269.3$ & $133.3 \pm 150.4$ & $40-160$ \\
CPK $(\mathrm{U} / l)$ & $186.4 \pm 36.5$ & $195.4 \pm 50.0$ & $130-250$ \\
Total cholesterol $(\mathrm{mg} / \mathrm{dl})$ & $105.1 \pm 56.0$ & $131.2 \pm 71.3$ & $80-160$ \\
Triglyceride $(\mathrm{mg} / \mathrm{dl})^{\mathrm{b}}$ & $80.9 \pm 26.0$ & $95.3 \pm 17.3$ & $76-132$ \\
Prothrombin time $(\%)^{\mathrm{a}}$ & $348.7 \pm 123.8$ & $331.6 \pm 99.2$ & $152-260$ \\
Fibrinogen $(\mathrm{mg} / \mathrm{dl})$ & $19.8 \pm 19.4$ & $18.9 \pm 17.2$ & - \\
ESR (mm) 1 hour & $44.4 \pm 31.7$ & $43.2 \pm 28.0$ & - \\
\hline \multicolumn{1}{c}{2 hours } & & & \\
\hline
\end{tabular}

Values are mean $\pm \mathrm{SD} .{ }^{\mathrm{a}} ; \mathrm{p}<0.001,{ }^{\mathrm{b}} ; \mathrm{p}<0.01,{ }^{\mathrm{c}} ; \mathrm{p}<0.05$, respectively, by $t$-test. GOT: glutamic oxaloacetic transaminase, CPK: creatine phosphokinase, ESR: erythrocyte sedimentation ratio.
Table 3. Neurological Findings in Embolic Stroke and Non-Embolic Stroke

\begin{tabular}{|c|c|c|c|c|c|c|}
\hline \multirow[b]{3}{*}{ Level of consciousness } & \multicolumn{3}{|c|}{$\begin{array}{l}\text { Embolic stroke } \\
\qquad(\mathrm{n}=92)\end{array}$} & \multicolumn{3}{|c|}{$\begin{array}{l}\text { Non-embolic stroke } \\
\qquad(\mathrm{n}=107)\end{array}$} \\
\hline & \multirow[t]{2}{*}{ yes } & \multicolumn{2}{|r|}{ unknown } & \multirow[t]{2}{*}{ yes } & \multicolumn{2}{|r|}{ unknown } \\
\hline & & & & & & \\
\hline Alert & 10 & $(10.9)$ & & 33 & $(30.8)$ & \\
\hline Confusion & 39 & $(42.4)$ & & 57 & (53.3) & \\
\hline Somnolent & 34 & $(37.0)$ & & 16 & $(15.0)$ & \\
\hline Coma & 9 & $(9.8)$ & & 1 & $(0.9)$ & \\
\hline Headache & $16 / 72$ & $(22.2)$ & 20 & $16 / 93$ & $(17.2)$ & 14 \\
\hline Vomiting $^{\mathrm{a}}$ & $16 / 80$ & $(20.0)$ & 12 & $4 / 99$ & $(4.0)$ & 8 \\
\hline Convulsion $^{\mathrm{c}}$ & $4 / 76$ & $(5.3)$ & 16 & 0/98 & $(0.0)$ & 9 \\
\hline Incontinence $^{\mathrm{a}}$ & $25 / 72$ & $(34.7)$ & 20 & $15 / 100$ & $(15.0)$ & 7 \\
\hline Cervical bruit ${ }^{\mathrm{a}}$ & $4 / 92$ & $(4.3)$ & 0 & $18 / 107$ & $(16.8)$ & 0 \\
\hline Aphasia & $31 / 71$ & $(43.7)$ & 21 & $41 / 94$ & (43.6) & 13 \\
\hline Conjugate deviation ${ }^{\mathrm{a}}$ & $50 / 92$ & $(54.3)$ & 0 & $25 / 107$ & $(23.4)$ & 0 \\
\hline Anisocoria $^{\mathrm{a}}$ & $23 / 92$ & $(25.0)$ & 0 & $4 / 107$ & $(3.7)$ & 0 \\
\hline Horner's syndrome & 15 & & & 2 & & \\
\hline III palsy & 8 & & & 2 & & \\
\hline Absent light reflex ${ }^{b}$ & $8 / 92$ & $(8.7)$ & 0 & $1 / 107$ & $(0.9)$ & 0 \\
\hline VII palsy ${ }^{\mathrm{a}}$ & $77 / 85$ & $(90.6)$ & 7 & $81 / 104$ & (77.9) & 3 \\
\hline XII palsy ${ }^{\mathrm{a}}$ & $38 / 53$ & $(71.7)$ & 39 & $34 / 90$ & $(37.8)$ & 17 \\
\hline Motor paresis & $88 / 92$ & $(95.7)$ & 0 & $102 / 107$ & (95.3) & 0 \\
\hline Sensory disturbance ${ }^{a}$ & $57 / 65$ & $(87.7)$ & 27 & $44 / 91$ & (48.4) & 16 \\
\hline Pathological reflex ${ }^{c}$ & $71 / 92$ & $(77.2)$ & 0 & $67 / 106$ & $(63.2)$ & 1 \\
\hline
\end{tabular}

Numbers in parentheses are percentages. ${ }^{\mathrm{a}}$; $\mathrm{p}<0.01,{ }^{\mathrm{b}}$; $\mathrm{p}<0.02,{ }^{\mathrm{c}}$; $\mathrm{p}<0.05$, respectively, by chi-square test.
Table 5. CT Findings in Embolic Stroke and Non-Embolic Stroke

\begin{tabular}{|c|c|c|}
\hline & $\begin{array}{l}\text { Embolic stroke } \\
\qquad(\mathrm{n}=92)\end{array}$ & $\begin{array}{l}\text { Non-embolic stroke } \\
\qquad(\mathrm{n}=107)\end{array}$ \\
\hline \multicolumn{3}{|l|}{ 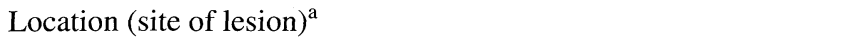 } \\
\hline No lesion & $3(3.3)$ & $4(3.7)$ \\
\hline Cortical* & $73(79.3)$ & $32(29.9)$ \\
\hline Subcortical & $14(15.2)$ & $24(22.4)$ \\
\hline Watershed area & $\begin{array}{ll}0 & (0.0)\end{array}$ & $39(36.4)$ \\
\hline Superficial & & 12 \\
\hline Deep & & 3 \\
\hline Superficial and deep & & 24 \\
\hline Unclassified cortical & $2(2.2)$ & $8 \quad(7.5)$ \\
\hline \multicolumn{3}{|l|}{ Brain edema $^{a}$} \\
\hline No edema & $10(10.9)$ & $39(36.4)$ \\
\hline Moderate $^{\dagger}$ & $36(39.1)$ & $53(49.5)$ \\
\hline Midline-shift & $43(46.7)$ & $11(10.3)$ \\
\hline \multicolumn{3}{|l|}{ Margin of hypodense area $^{a}$} \\
\hline Clear & $60(65.2)$ & $29(27.1)$ \\
\hline Unclear & $29(31.5)$ & $74(69.2)$ \\
\hline \multicolumn{3}{|l|}{ Hemorrhagic transformation $^{a}$} \\
\hline Within seven days of onset & $9(9.8)$ & $1(1.0)$ \\
\hline Total (during observation) & $38(41.3)$ & $5 \quad(4.7)$ \\
\hline Infarct Index $(\%)^{\mathrm{b}}$ & $30.6 \pm 23.9$ & $13.2 \pm 13.5$ \\
\hline
\end{tabular}

${ }^{*}$ Confined with vascular territories. ${ }^{\dagger}$ Deformity of the lateral ventricle or disappearance of the cortical sulci on the affected hemisphere is classified as moderate brain edema. Numbers in parentheses are percentages. Values are mean $\pm \mathrm{SD} .{ }^{\mathrm{a}} ; \mathrm{p}<0.01$ by chi-square test, and b; $<0.0001$ by $t$-test. 
of less than 0.05 was considered as statistically significant.

Preparation of a differentiation table to identify embolic from non-embolic stroke was attempted on the basis of the results obtained in the present study. In preparation of the quantitative table, 52 patients with EM and 66 patients with $\mathrm{NE}$ up to 1982 while the CT device was an EMI 1010 (Toshiba Co. Ltd., Tokyo) were applied. These patients were also used for an internal verification. External check was performed in 40 patients with EM and 41 patients with NE from 1983 when a TCT 20A (Toshiba Co. Ltd., Tokyo) was used.

The incidence of each category of items for the temporal profile, physical, neurological and CT findings were calculated and statistically compared between EM and NE using a chisquare test, as described in the previous section (Tables 2, 3, and 5). A preliminary discriminatory analysis was performed on items for which statistical significance was obtained, and a combination of those items that contribute to an improvement of the probability for correct diagnosis was determined. When some items were lacking due to insufficient information in the acute stage, they were categorized as "unknown". The system was simplified as much as possible to make evaluations of each item easier even for the general practitioner. The items and categories obtained were analyzed by Hayashi's quantification theory group II $(33,34)$.

\section{Hayashi's quantification theory group II}

When external bases (objective variables) are given as qualitative data in regression analysis, the discriminatory analysis is to be used as the statistical method which explains the external bases on the basis of quantitative items (explanatory variables). On the other hand, the statistical method appropriate to explain the external bases given qualitatively on the basis of qualitative items is the quantification theory group II (33). Application of this discriminatory function method using the categorical data as the variables is statistically optimum for preparation of a differentiation table.

The items (clinical features and CT findings) which were considered to influence the external base consisting of a group of $k$ ( $k=2$; EM and NE in the present study) are set. Then, suppose that the number of items is given as $m(m=11$, see Results) and the number of categories for each item as $n_{1}, n_{2}, \ldots$. , $n_{m}$. The values of case $s$ in group $k$ (EM or NE) relative to the $j$-th category of the $i$-th item may be expressed in the following dummy variable:

$$
\delta^{k s}{ }_{i j}=\left\{\begin{aligned}
1: & \text { when case } s \text { in group } k \text { responds to } j \text {-th category } \\
& \text { of } i \text {-th item, } \\
0: & \text { otherwise. }
\end{aligned}\right.
$$

Each item is assumed to respond to one category, but not to the other category. It may be assumed that these item-category data of each case can be obtained by a linear formula of the first degree:

$$
Y_{k s}=\sum_{i=1}^{m} \sum_{j=1}^{n i} \alpha_{i j} \delta^{k s}
$$

The coefficient $\alpha_{i j}$ (category weight) can be considered as the qualitative weight given to the item-category. Then, the category weight, $\alpha_{i j}$ is determined so that the weight, $Y_{k s}$ is small when the cases belong to the same group and large when they belong to different groups. This is equivalent to obtaining the category weight with the maximum correlation ratio $\left(\eta^{2}\right)$. The total variance $\left(\sigma_{T}^{2}\right)$ of $Y_{k s}$ is obtained from the sum of the between-group variance $\left(\sigma_{B}{ }^{2}\right)$ and the within-group variance $\left(\sigma_{W}^{2}\right)$. Therefore,

$$
1=\sigma_{B}^{2} / \sigma_{T}^{2}+\sigma_{W}^{2} / \sigma_{T}^{2}
$$

The correlation ratio $\left(\eta^{2}\right)$ is expressed as $\sigma_{B}^{2} / \sigma_{T}^{2}$, and the values are in the range 0 to 1 . Since $\sigma_{W}^{2}$ approaches 0 as $\eta^{2}$ approaches 1 , this means that the $k$ groups are separated.

The degree of influence of an item on the external bases is shown by the range of the category weights in the respective item. The degree of influence of each category in a given item on the external base is evident from the value of the category weight. In the same way as in regression analysis, it is possible to obtain the partial correlation coefficients between each item and external bases. The accuracy of the results of analysis is shown by the correlation ratio $\left(\eta^{2}\right)$ or the multiple correlation coefficient $(\eta)$.

\section{Results}

\section{Patients' baseline profiles}

Table 2 shows the clinical and laboratory findings for 92 patients with EM and 107 patients with NE. For EM, 79 patients (85.9\%) with potential cardiac sources of emboli were diagnosed as cardioembolic stroke. The respiration rate on admission was significantly higher in EM $(\mathrm{p}<0.01)$, while arterial blood pressure, both systolic and diastolic, was significantly higher in NE, $162.7 \pm 25.1 \mathrm{mmHg}$ and $89.6 \pm 14.7$, than in EM, $146.6 \pm 25.6$ and $85.1 \pm 14.8(\mathrm{p}<0.01)$. Previous transient ischemic attack (TIA) was found in $22.4 \%$ of NE, but in only $9.8 \%$ in EM. All TIAs in NE were stereotype, while more than one-half of TIAs in EM occurred in multiple vessels (Table 2). Abrupt onset of neurologic deficits was seen in $71.7 \%$ of EM, and many "unknown" cases with EM were found with a severe defect that suggested sudden onset with maximal neurologic deficits. An abrupt onset was reported in only $9.3 \%$ in NE. The time of onset in more than one-half of the patients in both groups was during daytime while active.

\section{Neurological findings}

The neurological findings in EM and NE are shown in Table 3. The level of consciousness on admission was more severely disturbed in EM in contrast to those in NE; i.e. somnolent in $37.0 \%$ and comatose in $9.8 \%$ in the former, and alert in $30.8 \%$ and confused in $53.3 \%$ in the latter $(\mathrm{p}<0.01)$. Patients with EM showed a significantly high incidence of vomiting and incontinence at onset $(\mathrm{p}<0.01, \mathrm{p}<0.01$, respectively). A cervical bruit was noted in 18 patients $(16.8 \%)$ in NE but in only four (4.3\%) in EM $(\mathrm{p}<0.01)$. No significant difference in the frequency of aphasia was observed between the two groups.

Conjugate eye deviation was significantly more common in 
EM $(54.3 \%)$ in comparison to NE $(23.4 \%)(\mathrm{p}<0.01)$. It was evident that hemispheric symptoms and signs were more extensive and severe in EM. Horner's syndrome was found in 15 patients $(16.3 \%)$ in EM but in only two (1.9\%) in NE. Thus, anisocoria in the acute stage was more frequently seen in EM than NE $(25.0 \%$ vs. $3.7 \%)(\mathrm{p}<0.01)$. Among abnormalities of the pupils, mydriasis associated with an absent pupillary light reflex due to oculomotor palsy was observed in eight patients (8.7\%) with EM, but in only two (1.9\%) with NE. Central VII nerve palsy, XII nerve palsy, sensory disturbance and Babinski's sign were significantly more common in EM than in NE ( $\mathrm{p}<0.01,0.01,0.01,0.05$, respectively).

\section{Laboratory examinations}

The results of routine laboratory examinations on admission are shown in Table 4. Hematocrit was significantly higher in NE $(\mathrm{p}<0.05)$, while the prothrombin time was significantly prolonged in EM $(p<0.001)$. However, the mean values of these variables were rather similar between the two groups and within the normal range, suggesting that these conventional parameters are not useful for identifying the pathophysiological mechanisms of stroke onset.

\section{Brain CT findings}

A summary of CT findings is shown in Table 5. A cortical hypodense area in a single vascular territory, a well marginated lesion, shift of midline structures (suggesting brain edema) and hemorrhagic transformation were significantly more prevalent in EM ( $<<0.01$, respectively). The infarct index was significantly greater in EM than in NE with mean values of $30.6 \%$ and $13.2 \%$, respectively $(\mathrm{p}<0.0001)$. When $\mathrm{CT}$ taken eight days after the onset was considered, hemorrhagic infarction was present in 38 patients with EM (41.3\%). Infarcts in the basal ganglia and/or deep white matter were present in 24 patients with NE (22.4\%) and 14 patients with EM (15.2\%). Watershed infarction on CT was found in 39 patients with NE (36.4\%); 22 with ICA occlusion and 17 with MCA stem occlusion. Of 39 watershed infarctions, 27 patients $(69.2 \%)$ showed the deep watershed infarction $(n=3)$ or the superficial and deep watershed infarction $(n=24)$. No watershed infarction was observed in EM.

\section{Quantitative differentiation table}

The category weights were calculated for each category of the respective 11 items which finally were selected by the quantification theory group II. The sample scores were $0.98 \pm$ 0.45 for $E M$ and $-0.77 \pm 0.53$ for NE. The difference in the mean values was 1.75 and the discriminatory point obtained from the cumulative distribution was 0.031 . There was a good discrimination between EM and NE as the external bases with a correlation ratio of $\eta^{2}=0.76$ and multiple correlation coefficient $R=0.87$.

The quantitative table was prepared by multiplying each category weight by 10 and rounding off the figures. Table 6 shows the numerical table (Embolic Score). When the total of scores in the 11 items was zero or greater, the patient was evaluated as EM, and when it was a negative number the evaluation was NE. The result of internal check revealed that the diagnostic accuracy was $98.9 \%$ for EM and $87.9 \%$ for NE. For the external verification, 40 patients with EM and 41 with NE during the year of 1983 and 1989 were studied. No significant differences were observed in any of the items in these two study periods (data not shown). With the external check, a favorable diagnostic accuracy of $90.0 \%$ for EM and $82.9 \%$ for NE were obtained.

Of 92 cases with EM, misdiagnosis by "Embolic Score" was found in five patients with mild neurologic deficits, who had a MCA branch occlusion. Diagnosis was incorrect in 15 of 107 cases with NE. Twelve of these had aphasia and disturbance of consciousness, suggesting the presence of extensive ischemia.

\section{Discussion}

In the present study, differences in temporal profile, physical, neurological and CT findings between EM and NE appear to depend on the pathophysiological mechanisms of each stroke subtype, i.e. the time course until cerebral arterial occlusion, the site of arterial occlusion and the state of the collateral circulation. Angiographic features of embolic stroke are as follows: 1) an abrupt arterial occlusion, 2) occlusion at the site just prior to arterial branching in the distal end of the ICA or MCA stem, or occlusion in the MCA branch, 3) absent or insufficient collateral circulation via the circle of Willis or leptomeningeal anastomoses, 4) frequent reopening phenomenon of the occluded vessel, and 5) frequent multiple arterial occlusions due to breakdown of the embolus $(2-8,26)$. The main radiographic features of thrombotic or hemodynamic stroke are as follows: 1) a gadual obstruction with thrombus formation on the atheromatous plaque, 2) an occlusion at the origin of ICA or MCA stem, 3) influence of hemodynamic factors such as grade of stenosis and/or collateral circulations via the circle of Willis or leptomeningeal anastomoses $(3,4,29)$.

A comprehensive clinical diagnosis of stroke subtypes is made in accordance with the temporal profile, neurological findings and the presence of cardiac disorders, systemic embolism or other risk factors, because there is no clinical "gold standard" for each stroke subtype other than pathological verification. Classification of stroke subtypes using $\mathrm{CAG}$ alone has some disadvantages, because an embolic stroke patient without reopening or an embolus shadow can potentially be diagnosed as NE. In clinical practice, however, conventional CAG can demonstrate the mechanisms of cerebral arterial occlusion more accurately than any other clinical tools (25), although Duplex carotid ultrasonography, transcranial Doppler ultrasonics, and magnetic resonance angiography are taking the place of this invasive examination. Thus, we used CAG for classification of the pathophysiological mechanisms to clarify and determine the differences in clinical features and CT findings.

\section{Clinical features and neurological findings}

With respect to the mode of onset, a progressive (insidious 
and stepwise) course was observed in $76.5 \%$ of NE, whereas $71.7 \%$ of patients with EM showed an abrupt onset. According to the statistical analysis used in the present study, the most useful item in discrimination between EM and NE was the rapidity of onset, as shown in Table 6. This indicates that the differences in the temporal profiles in the acute phase between EM and NE do not reflect the patients' background factors such as risk factors but rather they reflect the process of arterial occlusion. On the other hand, the differences in the respiration rate, vomiting, incontinence, consciousness disturbance, motor weakness and sensory disturbances between EM and NE can be

Table 6. "Embolic Score" for Identifying Embolic Stroke from Non-Embolic Stroke

1. Manner of onset

2. Vomiting at onset

3. Incontinence at onset

5. Cervical bruit

6. Anisocoria

7. Deviation of the tongue

8. Sensory disturbance

10. Brain edema on CT

No

Yes

No

Unknown

Cortical

Deep seated

Watershed

Miscellaneous

None

No edema

Moderate

Midline-shift explained mainly by the extent of infarct and the severity of brain ischemia.

Ocular symptoms and signs are also quite important for differential diagnosis in the acute stage of ischemic stroke. Conjugate deviation was found significantly more frequent in EM than NE, compatible with the more extensive and severe ischemia in EM. Anisocoria in the acute stage is observed in the presence of uncal (tentorial) herniation or Horner's syndrome on the affected side, and was significantly more common in EM. Horner's syndrome in cerebrovascular disease is known to be caused by brain stem infarction, but there have been a few detailed studies concerning supratentorial brain infarction in the internal carotid arterial system (35). The postganglionic fibers of the superior cervical sympathetic ganglia are divided into those which run upwards along the external carotid artery, controlling facial perspiration and vasomotor function, and those wich run upwards around ICA, controlling the pupils, eyelids and perspiration of the forehead (35). The reason for a high incidence $(16.3 \%$ ) of partial Horner's syndrome in EM may be sudden damage (or ischemia) to the local postganglionic fibers when the embolus passes through or obstructs ICA. 0 It is also suggested that the presence of supratentorial infarct lesions which expand towards the ipsilateral thalamus is one of the causative mechanisms for Horner's syndrome.

\section{Laboratory examinations}

The hematocrit was greater in NE than EM, suggesting that rheological factor may have been partly related to the development of non-embolic stroke $(36,37)$. However, these conventional laboratory studies were not useful for identifying embolic from non-embolic stroke, because differences in the mean value of each variable were close and within the normal range. Our earlier study demonstrated that measurement of hemostatic molecular markers, such as thrombin-antithrombin III complex and D-dimer are useful to differentiate with high accuracy cardioembolic stroke from the other stroke subtypes in the hyper acute stage (38), although such novel molecular markers are not generally available in clinical practice at present.

\section{Brain CT findings}

Cortical infarction confined to a vascular territory, shift of midline structure and hemorrhagic transformation on CT were observed in EM with a significantly high incidence, and these results are consistent with the previous observations (15-18, 30-32). A well-marginated cortical infarction confined to a vascular territory appears to reflect a distinct difference between infarcted and preserved brain tissue, since the ischemic injury in the embolic mechanism matches the territory of a cerebral artery $(16,18)$. However, the frequency of hemorrhagic transformation detected by $\mathrm{CT}$ is not very high within seven days of onset and requires very frequent and careful examination. Diagnostic accuracy is improved when hemorrhagic infarctions are detected after seven days and added to "Embolic Score".

Watershed infarction is known to be caused by atherothrombotic ICA occlusion with hemodynamic compro-

Thrombotic or Hemodynamic Stroke. 


\section{Embolic Score in Acute Ischemic Stroke}

mise, although the pathophysiology remains unclarified (30, 39-43). Among 39 cases with watershed infarction in the present series, MCA stem occlusion was found in 17 cases as well as 22 cases of ICA occlusion in NE. The evidence suggests that watershed infarction is caused not only by hemodynamic mechanism but also by multiple factors such as embolism, multiple microemboli or blood coagulation disorders $(41,43)$. Infarcts involving the deep watershed area were present in 27 of $39 \mathrm{NE}$ cases $(69.2 \%)$. We found that an infarction in the deep watershed area was characteristic of atherothrombotic brain infarction, while a superficial watershed infarction was also seen in $1.5 \%$ of embolic stroke in our another series (18).

\section{Quantitative differentiation table}

Numerical tables are widely used in clinical practice or research to diagnose stroke subtypes or to grade the severity of the illness (20-24), although some quantitative diagnostic criteria are not based on statistics. To prepare a numerical table for differential diagnosis, we used the quantification theory group II, because the external bases given qualitatively should be explained on the basis of qualitative factors, as mentioned in Methods (33, 34). "Embolic Score" derived in this study, showed a high diagnostic accuracy beyond $80 \%$ with internal and external verifications, and appeared to be useful for actual quantitative diagnosis. In EM, only five cases $(5.4 \%)$ were totally misdiagnosed. On the other hand, 15 cases $(14.0 \%)$ with NE who showed hemispheric syndromes, such as aphasia or consciousness disturbance were misdiagnosed as EM. Possible reasons are as follows: 1) diversification of the ischemic lesion depending on the mode of atherosclerotic arterial occlusion and the development of collateral circulations, 2) inclusion of thrombotic stroke patients with sudden arterial occlusion, such as rupture of atheromatous plaque, and 3) the presence of arteryto-artery embolism peripheral to the site of occlusion. Thus, the pathophysiology and pathogenetic mechanisms of thrombotic and hemodynamic strokes are much more heterogeneous. It is well known that atherothrombotic ICA occlusion may occur in the setting of acute stroke or may occur gradually or silently, depending on the intracranial collateral circulation (29).

\section{Conclusion}

From the present study, it was evident that the differences in clinical features and CT findings between EM and NE do not reflect the patients' backgrounds, rather they reflect the pathophysiological mechanisms of cerebral arterial occlusion at onset of stroke, that regulate the extent and severity of the ischemic lesion. Thus, investigations of therapeutic strategies during the acute stage based on the pathophysiological mechanisms may be more important than clinical categories, because clinical subtypes include various mechanisms of stroke onset. The "Embolic Score" derived from this study with a high diagnostic accuracy might also be helpful in investigations of acute ischemic stroke.

Acknowledgments: We thank Professor Marc Fisher, the Medical Center of Central Massachusetts and the University of Massachusetts Medical School, for his critical comments and review of the manuscript.

Supported in part by a research grant for cardiovascular diseases 62A-2, 9A-2 and 9A-8 from the Ministry of Health and Welfare, Tokyo, Japan.

\section{References}

1) National Institute of Neurological Disorders and Stroke Ad Hoc Committee. Classification of cerebrovascular disease III. Stroke 21: 637, 1990.

2) Mohr JP, Caplan LR, Melski JW, et al. The Harvard cooperative stroke registry: A prospective registry. Neurology 28: 754, 1978.

3) Yamaguchi T, Minematsu K, Choki J, Ikeda M. Clinical and neuroradiological analysis of thrombotic and embolic cerebral infarction. Jpn Circ J 48: 50, 1984.

4) Mohr JP, Barnett HJM. Classification of ischemic stroke. in: STROKE. Pathophysiology, Diagnosis, and Management, Barnett HJM, Mohr JP, Stein BM, Yatsu FM, Eds. Churchill Livingstone, New York, 1986, p. 281.

5) Timsit SG, Sacco MS, Mohr JP, et al. Early clinical differentiation of cerebral infarction from severe atherosclerotic stenosis and cardioembolism. Stroke 23: 486, 1992.

6) Cerebral Embolism Task Force. Cardiogenic brain embolism. Arch Neurol 43: 71, 1886.

7) Ramirez-Lassepas M, Cipolle RJ, et al. Can embolic stroke be diagnosed on the basis of neurologic clinical criteria? Arch Neurol 44: 87, 1987.

8) Timsit SG, Sacco RL, Mohr JP, et al. Brain infarction severity differs according to cardiac or arterial embolic source. Neurology 43: 728, 1993.

9) Moseley ME, Cohen Y, Mintorovitch J, et al. Early detection of regional cerebral ischemia in cats: comparison of diffusion- and T2-weighted MRI and spectroscopy. Magn Reson Med 14: 330, 1990.

10) Mintorovitch J, Moseley ME, ChileuittL, Shimizu H, Cohen $Y$, Weinstein PR. Comparison of diffusion- and T2-weighted MRI for the early detection of cerebral ischemia and reperfusion in rats. Magn Reson Med 18: 39, 1991.

11) Minematsu K, Li L, Fisher M, Sotak CH, Davis MA, Fiandaca MS. Diffusion-weighted magnetic resonance imaging: rapid and quantitative detection of focal brain ischemia. Neurology 42: 235, 1992.

12) Warach $\mathrm{S}$, Chien $\mathrm{D}$, Li W, Ronthal M, Edelman RR. Fast magnetic resonance diffusion-weighted imaging of acute human stroke. Neurology 42: 1717, 1992.

13) Warach S, Gaa J, Siewert B, Wielopolski P, Edelman RR. Acute human stroke studied by whole brain echo planar diffusion-weighted magnetic resonance imaging. Ann Neurol 37: 231, 1995.

14) Takano K, Latour LL, Formato JE, et al. The role of spreading depression in focal ischemia evaluated by diffusion mapping. Ann Neurol 39: 308, 1996.

15) Lodder J. CT-detected hemorrhagic infarction; relation with the size of the infarct, and the presence of midline shift. Acta Neurol Scand 70: 329, 1984.

16) Weisberg LA. Computerized tomographic findings in cardiogenic cerebral embolism. Comput Radiol 9: 189, 1985.

17) Hart RG, Easton JD. Hemorrhagic infarcts. Stroke 17: 586, 1986.

18) Takano K, Minematsu K, Yamaguchi $T$, Sawada $T$, Omae T. Differences in CT findings between embolic and thrombotic cerebral artery occlusion of the internal carotid arterial system. Clin Neurol (Tokyo) 29: 1370, 1989 (Abstract in English)

19) von Kummer R. Early findings of computed tomography and angiography in acute stroke and thrombolytic therapy. in: Thrombolytic Therapy in Acute Ischemic Stroke III, Yamaguchi T, Mori E, Minematsu K, del Zoppo GJ, Eds. Springer, Heidelberg, 1995, p. 41.

20) Mahoney FI, Barthel DVY. Functional evaluation. Barthel index. Md State Med J 14: 61, 1965.

21) Folstein MF, Fostein SE, McHugh PR. "Mini-Mental State": A practical method for grading the cognitive state of patients for the clinician. J Psychiatr Res 12: 189, 1975.

22) Gottfries CG, Brane G, Steen G. A new rating scale for dementia 


\section{TAKANO et al}

syndrome. Gerontology 28 Suppl II: 20, 1982.

23) Loeb C. Vascular dementia: in: Handbook of Clinical Neurology. Vol.46, Neuro-behavioral Disorders, Vinken PJ, Bruyn GW, Eds. Elsevier, Amsterdam, 1985, p. 353.

24) Hachinski VC, Iliff LD, Zilkha E, et al. Cerebral blood flow in dementia. Arch Neurol 32: 632, 1975

25) Pessin MS, Hinton RC, Davis KR, et al. Mechanisms of acute carotid stroke. Ann Neurol 6: 245, 1979.

26) Minematsu K, Yamaguchi T, Omae T. 'Spectacular shrinking deficit': Rapid recovery from a major hemispheric syndrome by migration of an embolus. Neurology 42: 157, 1992.

27) Cerebral Embolism Study Group. Immediate anticoagulation of embolic stroke. A randomized trial. Stroke 14: 668, 1983.

28) Yamaguchi T, Minematsu K, Choki J. Arterial embolism. in: Cerebral Blood Flow: Physiologic and Clinical Aspects, Wood JH, Ed. McGrowHill Book Co., New York, 1987, p. 503.

29) Lownie SP, Lee DH, Fox AJ, Pelz DM. Cerebral angiography: in: STROKE. Pathophysiology, Diagnosis, and Management. Second Edition, Barnett HJM, Mohr JP, Stein BM, Yatsu FM, Eds. Churchill Livingstone, New York, 1992, p. 215.

30) Wodarz R. Watershed infarctions and computed tomography. A topological study in cases with stenosis or occlusion of carotid artery. Neuroradiology 19: 245, 1980.

31) Damasio H. A computed tomographic guide to the identification of cerebral vascular territories. Arch Neurol 40: 138, 1983.

32) Okada $Y$, Yamaguchi T, Minematsu K, et al. Hemorrhagic transformation in cerebral embolism. Stroke 20: 598, 1989.

33) Hayashi C. On the prediction of phenomena from quantitative data and the quantification of qualitative data from the methematico-statistical point of view. Ann Inst Statist Math 3: 69, 1952.

34) Tanaka $H$, Ueda $Y$, Hayashi $M$, et al. A quantitative diagnostic method for differentiating the subtypes of stroke in epidemiologic studies. Osaka City Med J 28: 31, 1982.

35) Millikan CH, McDowell F, Easton JD. Completed stroke: in: Stroke, Millikan CH, McDowell F, Easton JD, Eds. Lea \& Febiger, Philadelphia, 1987, p. 131.

36) Tohgi H, Yamanouchi H, Murakami M, Kameyama M. Importance of the hematocrit as a risk factor in cerebral infarction. Stroke 9: 369, 1978.

37) Kiyohara $Y$, Ueda K, Hasuo $Y$, et al. Hematocrit as a risk factor of cerebral infarction: long-term prospective population survey in a Japanese rural community. Stroke 17: 687, 1986.

38) Takano K, Yamaguchi T, Uchida K. Markers of a hypercoagulable state following acute ischemic stroke. Stroke 23: 194, 1992.

39) Jørgensen L, Torvik A. Ischemic cerebrovascular diseases in autopsy series: Part 2. Prevalence, location, pathogenesis and clinical course of cerebral infarcts. J Neurol Sci 9: 285, 1969.

40) Bogousslavsky J, Regli F. Unilateral watershed cerebral infarctions. Neurology 36: 373, 1986.

41) Ringelstein EB, Zeumer $H$, Angelou D. The pathogenesis of strokes from internal carotid artery occlusion. Diagnostic and therapeutical implications. Stroke 14: 867, 1983.

42) Torvik A, Skullerud K. Watershed infarcts in the brain caused by microemboli. Clin Neuropathol 1: 99, 1982.

43) Torvik A. The pathogenesis of watershed infarctions in the brain. Stroke 15: 221, 1984. 